\title{
ROTACIÓN DE CULTIVOS EN LA REGIÓN PAMPEANA: VOLVIENDO A LAS FUENTES ${ }^{\circ}$
}

\author{
CROP ROTATION IN THE PAMPEAN REGION: BACK TO THE SOURCES
}

Tomás Loewy*

enviado: 23 febrero 2017 - aceptado: 20 marzo 2017

\begin{abstract}
Resumen
Debido a los cambios en la actividad agropecuaria, palabras y conceptos, otrora estables, van mutando. La "rotación de cultivos" es una Buena Práctica Agrícola (BPA) emblemática en sistemas mixtos. El objetivo de este trabajo es rescatar su concepto agronómico, propiciando su adopción en escenarios de desarrollo local y cuidado del ambiente. Se presenta un esquema que diferencia monocultivo, secuencia, pradera y rotación, con sus respectivos impactos sobre la calidad del suelo. Las tecnologías nunca son neutrales y para calificarlas hay que ubicar lo agrario dentro de lo nacional/global, en períodos de tiempo abarcativos. La decantación de técnicas de procesos y de insumos es parte del diseño de sistemas y de la sustentabilidad de los territorios.
\end{abstract}

Código JEL: Q16

Palabras clave: rotaciones, buenas prácticas, sistemas, ambiente.

\begin{abstract}
Due to the changes in agricultural activity, words and concepts that used to be stable are now mutating. "Crop rotation" is a Good Agricultural Practice (GAP), emblema-

* Universidad Nacional del Sur, Bahía Blanca, Argentina. Correo electrónico: tomasdarre@gmail.com. Actualización del trabajo presentado en actas III Jornadas Interdisciplinarias del Sudoeste Bonaerense. UNS. Bahía Blanca. (2004): 49-52.

- Loewy, T. (2017). Rotación de cultivos en la región pampeana: volviendo a las fuentes. Estudios económicos, 34 (68), 93-99.
\end{abstract}


tic in mixed systems. The objective of this work is to rescue its agronomic concept, favoring its adoption in local development and environmental care scenarios. A scheme that differentiates monoculture, crop sequence, meadow and rotation, with their respective impacts on soil quality, is presented. Technologies are never neutral, and in order to qualify them, it is necessary to locate the agrarian question within the national / global context in encompassing time periods. The decantation of both process and input techniques is part of the systems design and the sustainability of the territories.

JEL Code: Q16.

Keywords: Rotations, Good Practices, Systems, Environment.

\section{INTRODUCCIÓN}

La dinámica de la actividad agropecuaria, en los últimos años, va mutando palabras y conceptos, otrora estables. Tal es el caso de lo que nos enseñaron en las facultades como "rotación de cultivos", acepción hoy un tanto devaluada. El "redescubrimiento" de esta clásica y milenaria práctica agronómica apareció, frente a la expansión lineal de la soja, a principios de este siglo.

Alternando esta oleaginosa con cereales de invierno y/o de verano se mejora el balance de la materia orgánica del suelo y el control de ciertas plagas. La fertilización también puede hacer una contribución positiva. Estos presupuestos, sin embargo, no son suficientes para sostener la viabilidad ecológica de este dispositivo técnico. La sustentabilidad, bien entendida, invoca la multifuncionalidad del sistema productivo en sus atributos sociales, ambientales y económicos, en forma simultánea. Al respecto resulta promisorio que la organización del premio Nobel haya instituido este año la categoría Desarrollo Sustentable, invocándolo como una condición de supervivencia de la humanidad ${ }^{1}$.

Este contexto conceptual es necesario si queremos adjudicar un significado unívoco a los términos agronómicos para mejorar la comunicación y el diseño de unidades apropiadas en cada territorio. Confundir secuencias con rotaciones o agricultura con agronegocio acarrea, a la larga, consecuencias indeseables para toda la sociedad.

Dado que la "rotación de cultivos" se presenta como una Buena Práctica Agrícola (BPA), emblemática en sistema mixtos, el objetivo de este trabajo es rescatar su concepto agronómico, propiciando su adopción en escenarios de desarrollo local y cuidado del ambiente.

1 This Nobel Peace Prize themed for Sustainable Development, 2017, http://np4sd.org/ 


\section{MONOCULTIVO, SECUENCIA Y ROTACIÓN}

Dos o más cultivos anuales, distintos y sembrados uno tras otro, conforman una secuencia con innegables ventajas sobre el monocultivo. No debemos confundirlo, sin embargo, con el concepto agronómico original que - en su definición - contempla la alternancia de distintas siembras anuales (en secuencia) con praderas perennes. Estos dos módulos se suceden, en forma programada, dentro de los distintos lotes que conforman la unidad predial. Las opciones básicas posibles, en los sistemas de producción, se resumen en el siguiente esquema.

Tabla 1. Patrones agronómicos de producción extensiva entre distintos tipos de cultivos

\begin{tabular}{cccc}
\hline Sistema & Cultivo & Característica & Rubro \\
\hline Monocultivo & Anual & Una especie & Agrícola o ganadero \\
Secuencia & Anual & Varias especies & $\begin{array}{c}\text { Agrícola y/o } \\
\text { ganadero }\end{array}$ \\
Pradera & Plurianual & Una o más especies & Ganadería \\
Rotación & Anual y plurianual & En alternancia & Mixto \\
\hline
\end{tabular}

Fuente: elaboración propia.

El sistema mixto requiere que la agricultura y la ganadería se integren dentro de los mismos lotes, sin implicar - necesariamente - una rotación de cultivos. La alternancia de cultivos anuales y perennes aporta a las componentes de sustentabilidad ambiental y económica, más allá del corto plazo. Su promoción, junto a otras BPA, se puede transformar en una herramienta de política activa para viabilizar pymes agropecuarias (Loewy et al., 2015).

Las distintas secuencias de cultivos estacionales, de invierno o de verano, para forraje o para grano, no son mejoradoras de la calidad del suelo. La diferencia cualitativa entre secuencias y rotaciones, radica en la construcción de fertilidad edáfica de las praderas y su posterior aprovechamiento por los cultivos anuales (Loewy y López, 1994; Casanovas, Echeverría y Studdert, 1995).

La inclusión de leguminosas anuales en una secuencia, por ejemplo, vicia consociada en verdeos, mejora el balance de nitrógeno en el suelo, favoreciendo hasta dos cultivos siguientes. La soja, en cambio, presenta un doble balance negativo de materia orgánica y de nitrógeno (Loewy, 1987; Álvarez, Lemcoff y Merzari, 1995). Solo las praderas pueden mejorar sustancialmente el recurso tierra, bajo ciertas condiciones de utilización. 


\section{SIEMBRA DIRECTA, SOJIZACIÓN Y ROTACIONES}

El advenimiento de la siembra directa (SD) hace varias décadas desdramatizó la agricultura continua pero no el monocultivo. Esta técnica se puede relacionar con el control de la erosión eólica y una mejora en la fracción orgánica del suelo, entre otros atributos. Asociada a un paquete tecnológico cerrado que incluye el monocultivo, la soja transgénica $(\mathrm{MG})^{2}$ y el herbicida Glifosato - en cambiocontribuyen a un modelo altamente insustentable.

Tal fue el caso de lo que dio en llamarse "sojización", por la evolución lineal de esta leguminosa bajo una modalidad con altos impactos negativos para el país. Hacia 1970 la superficie de la soja fue de 95.650 hectáreas, llegando a 6 millones en 1996. A partir de ese año su crecimiento fue más acelerado aun, cuando se aprobó la liberación al mercado de la soja MG, oscilando actualmente en los 20 millones de hectáreas (Ghida Daza, 2002; Bolsa de cereales, 2016).

Al comienzo del siglo hubo un alza significativa del valor de los granos a nivel global, especialmente de la soja. Esto dio lugar a un período muy favorable para la generación de bienes primarios, con producción de agrocombustibles y una demanda consolidada de China e India. En América esto funcionó como un nuevo consenso, en este caso de los commodities, ratificando la división internacional del trabajo (Svampa, 2013).

Paralelamente, operó una prevalencia de la agricultura industrial como sistema de producción, homogeneizante, en detrimento de la agricultura familiar, diversa (Gudynas, 2008). La biotecnología, por otra parte, ejemplificada en la transgénesis, en conjunto hizo un aporte negativo a la sustentabilidad de los sistemas agrícolas (Marsden, 2008).

En resumen, el proceso de sojización no solucionó el problema económico, a la par que dejó pasivos ambientales y sociales muy relevantes, aun cuando resulta escasa la mención pública y mediática de esta problemática, que es estructural y global (Florio, 2017). Los gobiernos de la región privilegiaron el corto plazo sin promover otros cultivos y sistemas para neutralizar el monocultivo, la tala de bosques y contaminación. Solo al comienzo algunos referentes técnicos y corporativos, mencionaron la "rotación" de cultivos (omitiendo las praderas) como un "solución" al problema ambiental.

2 Una semilla modificada genéticamente por la empresa Monsanto para resistir la aplicación de un potente herbicida. 


\section{USO DEL SUELO, TEMPORALIDAD Y SISTEMAS}

Los suelos albergan millones de años, desde su génesis, a partir de la "roca madre". Finalmente, alcanzan una fertilidad climáxica, en equilibrio semiestable con el ambiente. La aparición reciente, en tiempos geológicos, de la agricultura y la ganadería (acción antrópica) tornó más inestable el recurso y más expuesto a las fuerzas erosivas del viento y del agua. La rotación de cultivos, en el sentido que aquí le otorgamos, es uno de los mecanismos consistentes para restablecer un nuevo equilibrio en la fertilidad del suelo. Esta y otras tecnologías de procesos, complementada con los insumos necesarios, configuran elementos sensibles de un sistema de producción.

El modelo actual, prevaleciente, desdeña los costos sociales y ambientales de la producción. Si los incluyera, la rentabilidad relativa de cultivos y sistemas variaría significativamente. La diferencia se llama sustentabilidad, y quedan pocos atajos para no abordarla con rigor, en la Argentina y en el mundo (Loewy, 2016a).

El enfoque de sistemas es una herramienta esencial del pensamiento moderno o del pensamiento, a secas (Senge, 1998; Capra, 2000). En el ámbito agropecuario, el sistema productivo es la mínima unidad crítica para evaluar su performance y una incumbencia insoslayable de la profesión agronómica (Loewy, 2009 y 2016b). El productivismo, sin concesiones, es una simplificación que colisiona con la vida. Paradójicamente, o no, aún se invocan razones humanitarias para sostener esa prédica.

Los impactos del cambio climático son crecientemente más graves y destructores, mientras que la temperatura atmosférica está aumentando más rápido de lo previsto. La siguiente revolución productiva será, necesariamente, una economía baja en el uso de recursos y de carbono (Programa de las Naciones Unidas para el Medio Ambiente y Sustainlabour, 2011).

La "rotación de cultivos" en muchas zonas, incluido el sudoeste bonaerense, podría ser un ícono de avanzada para incorporar un paradigma agrosocial o agroecológico (multifuncional), en sustitución del agro industrial (monofuncional). Teniendo en cuenta - además - que esta práctica es uno de los recursos primordiales hacia la gestión del carbono en el suelo, la Argentina puede contribuir mejor a las metas fijadas por el COP21 (París/2015) para mitigar el cambio climático³.

3 Existe una iniciativa global específica en ese sentido, "La iniciativa 4 por 1000", en agriculture. gouv, http://agriculture.gouv.fr/sites/minagri/files/1509-climat-4pour1000-esp_nov2015.pdf 


\section{CONCLUSIONES}

El ejemplo de la soja y las acepciones de la rotación de cultivos sirve para ejemplificar los riesgos de un enfoque sectorial y atemporal de la producción primaria. Las tecnologías nunca son neutrales, pero para calificarlas hay que ubicar lo agrario dentro de lo nacional/global y en espacios de tiempo abarcativos. Sin esa sintonía es difícil asociar el significante (nombre) de algo con la representación o el concepto. Aquí teníamos dos significados para un mismo significante y es lo que se trató de superar en este escrito, diferenciando "rotación" y "secuencia" de cultivos.

La decantación de tecnologías de procesos y de insumos es una parte del diseño de sistemas que brinden sustentabilidad a los territorios. Este requisito aparece como esencial dentro del dramático cambio global que estamos experimentando. En esa línea, dentro de la universidad serían deseables currículos académicos más acordes a estos temas y desde el Estado, políticas activas para incorporar los aspectos ambientales y sociales al complejo agroalimentario.

\section{BIBLIOGRAFÍA}

Álvarez, R., Lemcoff, J. H. \& Merzari, A. H. (1995). Balance de nitrógeno en un suelo cultivado con soja. Ciencia del Suelo, 21 (1), 38-40.

Bolsa de cereales (2016). Informe de Pre-Campaña No 18. Estimaciones Agrícolas. (Buenos Aires, 13 de octubre).

Casanovas, E. M., Echeverría, H. E. \& Studdert, E. A. (1995). Materia orgánica del suelo bajo rotaciones de cultivos. II efecto de los ciclos de agricultura y pastura. Ciencia del Suelo, 13, 21-27.

Capra, F. (2000). La trama de la vida. Una nueva perspectiva de los sistemas vivos. Barcelona: Anagrama.

Ghida Daza, C. (agosto, 2002). Área Economía, Estadística Informática. Informe Técnico. Marcos Juárez, Córdoba: Instituto Nacional de Tecnología Agropecuaria.

Florio, L. (2017). Agua y fuego sobre los campos argentinos. El modelo transgénico nuevamente en cuestión. Revista Criterio. Sección Debates, N. 2434. Recuperado de http://feedproxy.google.com/ r/RevistaCriterio/ 3/1_cbp$\mathrm{Db} 4 \mathrm{puE} /$ ?utm_source=feedburner\&utm_medium=email

Gudynas, E. (2008). Un análisis preliminar de las transformaciones recientes en la agricultura Latinoamericana. Chile. Economía Crítica \& Desarrollo, 3 (5), 167-191.

Loewy, T. (2016a). Modelos agrarios, suelos y sustentabilidad: un análisis cualitativo. Actas XXV Congreso Argentino Nacional de Suelos. Comisión 4, tra- 
bajo Nro. 361. Río Cuarto, Córdoba: Universidad Nacional de Río Cuarto. Recuperado de http://www.proyectodepais.com.ar/?p=460

Loewy, T. (2016b). Sistemas de producción y competencias agronómicas. Revista Agrouns, 13 (25), 12-14. Recuperado de http://servicios.uns.edu.ar/institucion/files/1AP087.pdf

Loewy, T. (2009). Sustentabilidad: hacia la recuperación de un perfil operativo. Actas IV Jornadas de la Asociación Argentino Uruguaya de Economía Ecológica. Buenos Aires, Argentina. Recuperado de http://inta.gob.ar/sites/default/files/script-tmp-1_sustentabilidad.pdf

Loewy, T. (1987). Rotación leguminosa-trigo y fertilidad nitrogenada del suelo. Ciencia del Suelo, 5 (1), 57-64.

Loewy, T., Milano, F. A., Ángeles, G. R., Saldungaray, M. C., Campaña, D. H. \& Alamo, M. A. (2015). Buenas prácticas agrícolas con desarrollo local para el sudoeste bonaerense. Serie Extensión. Colección Ciencias y Tecnología. Bahía Blanca: Ediuns. Recuperado de http://www.proyectodepais. com.ar/?p=392

Loewy, T. \& López, R. L. (1994). Efecto de pradera mixta y cultivos anuales sobre las propiedades de un Haplustol Entico. Ciencia del Suelo, 12 (7), 7-10.

Marsden, T. (2008). Agri-food contestations in rural space: GM in its regulatory context. Geoforum, 39 (1), 191-203.

Programa de las Naciones Unidas para el Medio Ambiente y Sustainlabour (2011). Cambio climático, sus consecuencias en el empleo y la acción sindical. Un

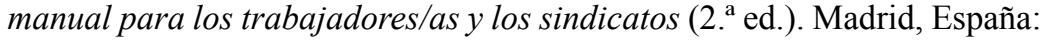
PNUMA/Sustainlabour.

Senge, P. M. (1998). La quinta disciplina. El arte y la práctica de la organización abierta al aprendizaje. México: Granica.

Svampa, M. (2013). Consenso de los Commodities y lenguajes de valoración en América Latina. Nueva Sociedad, (244), 30-46. Recuperado de http:// www.unesco.org.uy/shs/redbioetica/fileadmin/shs/redbioetica/Consenso_ de_Commodities.pdf

(C) 2017 por los autores; licencia otorgada a la revista Estudios económicos. Este artículo es de acceso abierto y distribuido bajo los términos y condiciones de una licencia Atribución-No Comercial 3.0 Unported (CC BY-NC 3.0) de Creative Commons. Para ver una copia de esta licencia, visite http://creativecommons.org/ licenses/by-nc/3.0/ 